\title{
POSIBLE SÍNDROME DE MÚLTIPLES MANCHAS BLANCAS EVANESCENTES RECURRENTE Y NEOVASCULARIZACIÓN COROIDEA
}

\section{POSSIBLE RECURRENT MULTIPLE EVANESCENT WHITE DOT SYNDROME AND CHROROIDAL NEOVASCULARIZATION}

\author{
FERNÁNDEZ-BARRIENTOS Y , DÍAZ-VALLE D², MÉNDEZ-FERNÁNDEZ R, \\ BENÍTEZ-DEL-CASTILLO JM ${ }^{2}$
}

\section{RESUMEN}

Caso clínico: Se presenta un caso de presunto síndrome de múltiples manchas blancas evanescentes (MEWDS) recurrente y bilateral (tres episodios bilaterales en nueve años). Trece años después del episodio inicial, presentó una membrana neovascular subretiniana (MNVSR) que respondió favorablemente a terapia fotodinámica, con una agudeza visual final de 20/40.

Discusión: El MEWDS se considera una enfermedad inflamatoria con buen pronóstico visual. Sin embargo, existe la posibilidad de desarrollar a largo plazo complicaciones como la MNVSR. Por ello, recomendamos el seguimiento periódico de estos pacientes por la importancia del diagnóstico y tratamiento precoz de las MNVSR en la agudeza visual final.

Palabras clave: Síndrome de múltiples manchas blancas evanescentes, recurrente, bilateral, neovascularización coroidea, tratamiento.

\section{ABSTRACT}

Case report: We present an unusual case of recurrent and bilateral multiple evanescent white dot syndrome (MEWDS), who had three bilateral episodes in a period of nine years. Thirteen years after the first episode, she was diagnosed with subretinal neovascularization (SRNV) which showed a satisfactory response to photodynamic therapy, with a final visual acuity of 20/40.

Discussion: MEWDS is considered classically to be an inflammatory disease with a good visual prognosis. Although the development of SRNV is unusual, we recommend long term follow-up of these patients since the early diagnosis and treatment of SRNV are both relevant for the retention of visual acuity (Arch Soc Esp Oftalmol 2007; 82: 587-590).

Key words: Multiple evanescent white dot syndrome, recurrent, bilateral, choroidal neovascularization, treatment.

\footnotetext{
Recibido: 3/8/06. Aceptado: 25/7/07.

Hospital Clínico Universitario San Carlos. Instituto de Investigaciones Ramón Castroviejo. Madrid. España.

1 Licenciado en Medicina.

2 Doctor en Medicina.

Correspondencia:

Yolanda Fernández Barrientos

C/. Vallehermoso, 15, 2. ${ }^{\circ}$, apto. 20

28015 Madrid

España

E-mail: yferbar@hotmail.com
} 


\section{INTRODUCCIÓN}

El síndrome de múltiples manchas blancas evanescentes (MEWDS) se considera una entidad de curso clínico autolimitado, benigna, de etiología desconocida y recurrencias infrecuentes. Sin embargo, la pérdida severa de visión secundaria a neovascularización coroidea ha sido descrita en la literatura $(1,2)$. Se presenta un caso de presunto MEWDS recurrente, que desarrolló una membrana neovascular subretiniana (MNVSR) 13 años después del episodio inicial.

\section{CASO CLÍNICO}

Mujer emétrope de 36 años, que acude a nuestro servicio de urgencias por visión borrosa sin episodio gripal previo en enero de 1992. A la exploración presentaba una agudeza visual (AV) en ojo derecho (OD) 20/30 y en el ojo izquierdo (OI) 20/60, células en cámara anterior de $1+\operatorname{ambos}$ ojos (AO) y múltiples puntos blancos de 100-200 micras más numerosos en el área temporal a la mácula en AO. La angiofluoresceingrafía (AFG) mostró múltiples puntos hiperfluorescentes (figs. 1 y 2). Se inició tratamiento con $40 \mathrm{mg}$ al día de metilprednisolona IM (Urbason ${ }^{\circledR}$ soluble $40 \mathrm{mg}$, Aventis Pharma S.A., Madrid), atropina sulfato $10 \mathrm{mg} / \mathrm{ml}$ tópica (Atropina ${ }^{\circledR} 1 \%$, Alcon Cusí S.A., Barcelona) cada 12 horas y dexametasona $1 \mathrm{mg} / \mathrm{ml}$ tópica (Maxidex ${ }^{\circledR}$, Alcon Cusí S.A., Barcelona) cada 6 horas. A las dos semanas de tratamiento alcanzó una AV de 20/20 en AO. Las pruebas sistémicas solicitadas (analítica básica,

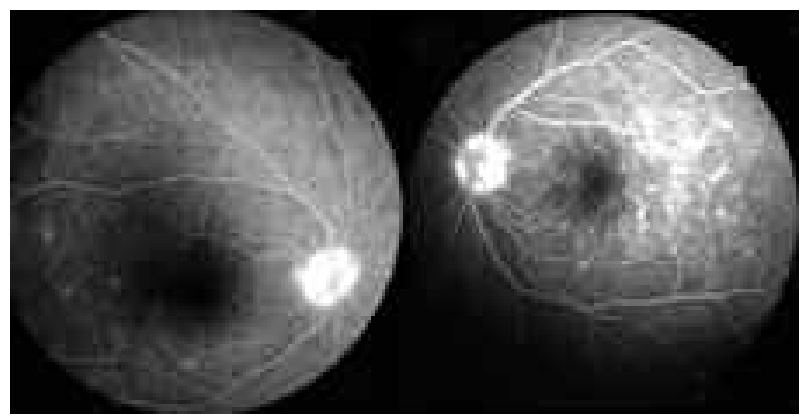

Figs. 1 y 2: Angiofluoresceingrafía en tiempos tardios del primer episodio de MEWDS bilateral, se observan múltiples puntos hiperfluorescentes más numerosos en la región temporal, describiendo un patrón en guirnalda característico del MEWDS; la papila muestra también hiperfluorescencia tardía con escape de colorante. radiografía de tórax, serología luética, enzima de conversión de la angiotensina y Mantoux) fueron negativas.

Cuatro años más tarde presentó una nueva recidiva, más acusada en OD, con pérdida visual al nivel de 20/60 OD y 20/30 OI, sin evidenciarse alteraciones campimétricas. Se inició la misma pauta sistémica que en el episodio anterior con recuperación visual completa al mes de tratamiento, persistiendo alguna mancha blanca y pigmento peripapilar temporal superior en el OD.

En septiembre de 2001 presentó un nuevo brote de carácter más leve. La AV era de 20/25 OD y 20/20 OI, apreciándose en la exploración funduscópica manchas blanco-amarillentas distribuidas por polo posterior y alteración del epitelio pigmentario retiniano (EPR) macular $\mathrm{AO}$. Se inició tratamiento corticoideo tópico y sistémico como en el primer episodio. A las tres semanas desde el inicio del brote, la AV disminuyó a 20/40 OD y a 20/60 OI, por lo que se decidió añadir metotrexate oral (Metotrexato Lederle ${ }^{\circledR}$, Whyeth, Madrid) a bajas dosis. Se solicitó estudio de HLA A29 que resultó negativo.

En abril de 2005, la paciente se mantenía asintomática, con AV en 20/30 OD y 20/25 OI. En el fondo de ojo, presentaba un levantamiento del neuroepitelio yuxtafoveolar nasal en el OD, la tomografía de coherencia óptica confirmó el diagnóstico de sospecha de una MNVSR (figs. 3 y 4). La paciente rechazó el tratamiento, aunque cinco meses después, con una AV en OD de 20/60 se inició tratamiento de la MNVSR (fig. 5), con terapia fotodinámica (Visudyne ${ }^{\circledR}$, Novartis Farmacéutica S.A., Bar-

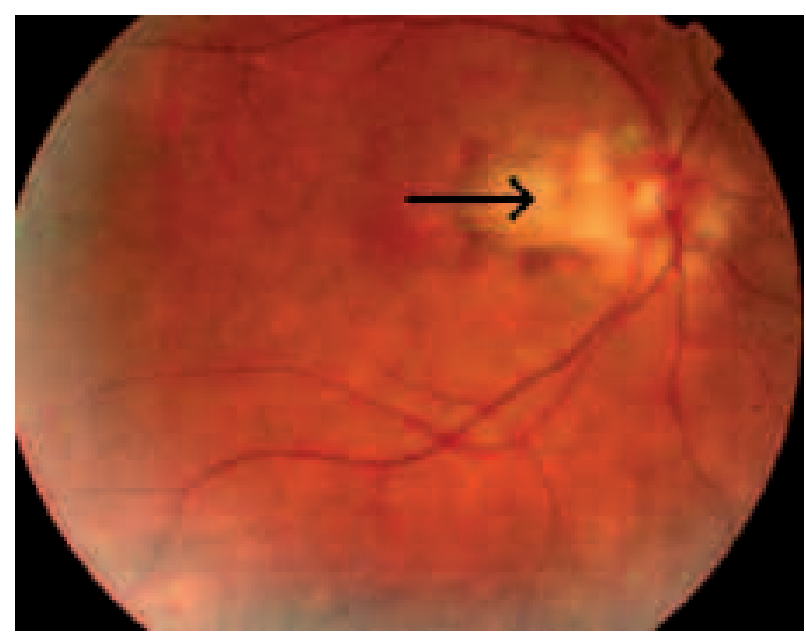

Fig. 3: Retinografía del OD, se observa el sangrado de la membrana neovascular subrretiniana. 


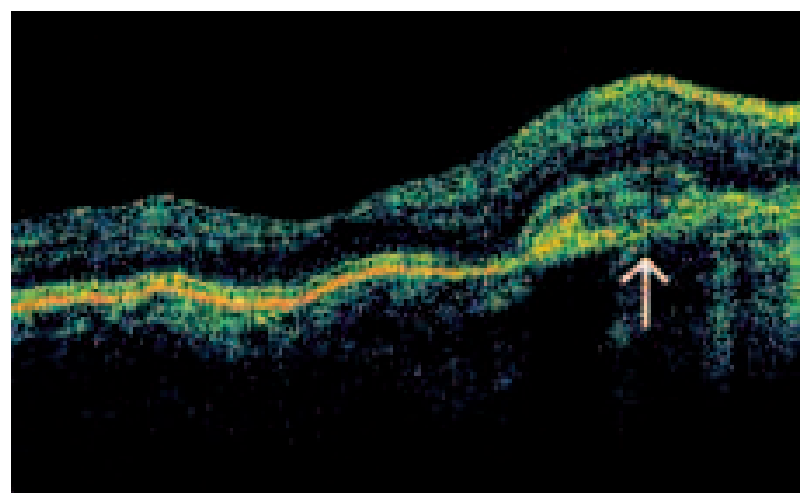

Fig. 4: Tomografía de coherencia óptica OD, corte horizontal (flecha negra en la figura 3). Membrana neovascular subretiniana de $200 \mu \mathrm{m}$ de grosor (flecha blanca).

celona) y triamcinolona acetónido intravitreo $4 \mathrm{mg}$ en $0,1 \mathrm{ml}$ (Trigón depot ${ }^{\circledR} 40 \mathrm{mg} / \mathrm{ml}$, Bristol-Myers Squibb, Madrid). La AV en el OD mejoró a 20/40”.

En el momento actual, la paciente presenta una AV 20/40 OD y 20/25 OI, manchas blanco-amarillentas tenues en polo posterior de ambos ojos, lesiones de atrofia peripapilar AO, con afectación del haz papilomacular en el OD y varias lesiones blanquecinas inactivas, inferiores a la mácula del OI (fig. 6).

\section{DISCUSIÓN}

El MEWDS es una enfermedad inflamatoria ocular infrecuente, de etiología desconocida, que suele afectar a mujeres jóvenes y en general presenta un

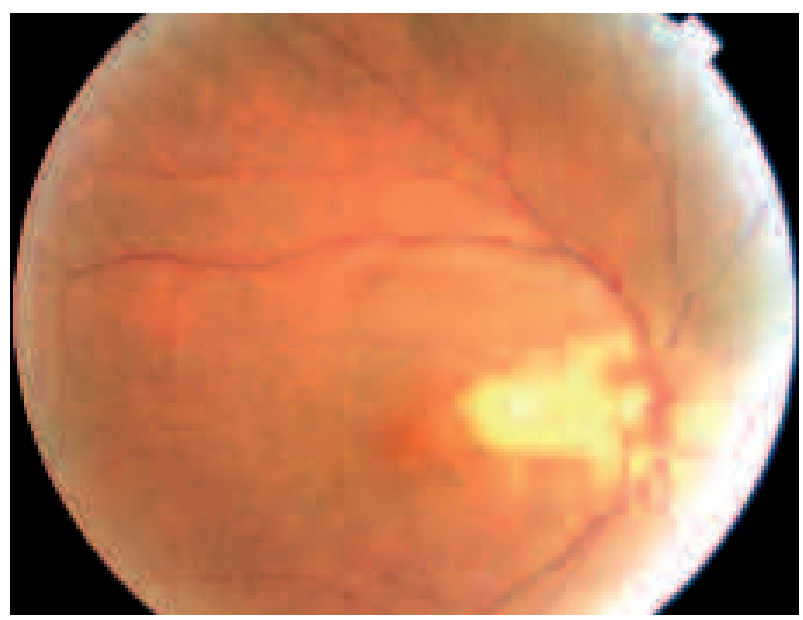

Fig. 5: Retinografía OD tras tratamiento con terapia fotodinámica, se observa un área de atrofia en al haz papilomacular y peripapilar.

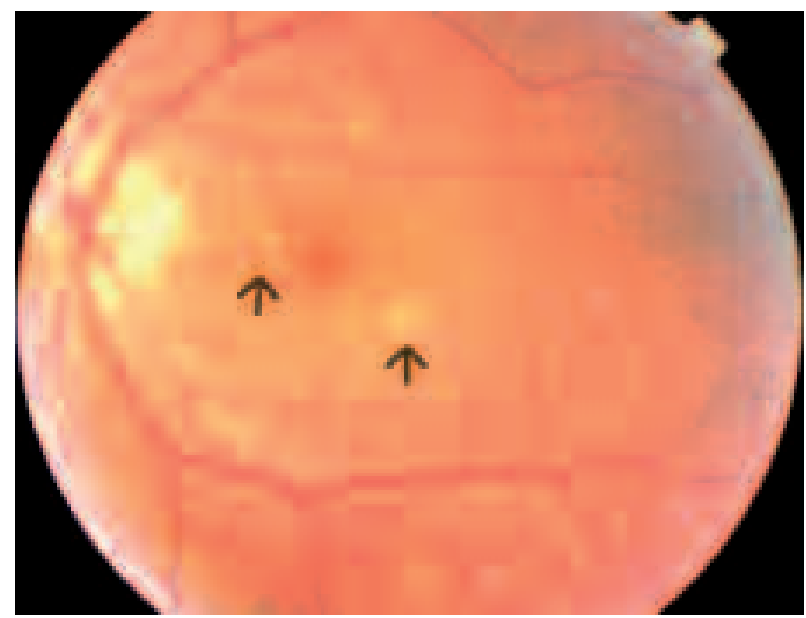

Fig. 6: Retinografía OI, se observas algunas manchas blancas tenues en polo posterior. Las flechas negras nos señalan dos lesiones blanquecinas de aspecto atrófico e inactivo.

curso autolimitado, con recuperación espontánea de la agudeza visual entre 3 y 10 semanas con mínimas secuelas en el EPR (1-5). En nuestro caso se documentaron tres episodios en 9 años, todos ellos bilaterales. Este patrón clínico de presentación del MEWDS (bilateral y recurrente) es muy infrecuente, se desconoce la razón por la que algunos pacientes presentan un curso diferente al resto $(2,3)$.

Hemos encontrado al menos cinco casos descritos en la literatura de MNVSR en pacientes con MEWDS (tabla I). En nuestro caso, la MNVSR se desarrolló 13 años después del primer brote de MEWDS siendo la AV final de 20/40.

En la evolución de MEWDS recurrente, la confluencia de áreas inflamatorias y la continuidad de la inflamación, pueden provocar cicatrices coriorretinianas (4). Nuestra paciente, desarrolló áreas de atrofia peripapilar y varias lesiones atróficas blancoamarillentas en áreas de antigua actividad inflamatoria. Estas alteraciones a nivel del EPR podrían predisponer al desarrollo de MNVSR.

En nuestro caso, las recurrencias de la enfermedad se controlaron con metotrexate a bajas dosis. La ciclosporina también ha sido utilizada con éxito para conseguir la remisión de los brotes (3).

Nuestra paciente no presentó vitritis, edema macular quístico, edema de papila, ni lesiones coriorretinianas periféricas características de la coroiditis multifocal (CMF). Además, era emétrope lo que descarta la coroidopatía puntata interna. Por otra parte, Kozielec et al (4) describen en pacientes 
Tabla I.

\begin{tabular}{|c|c|c|c|c|c|}
\hline Autores & $\begin{array}{l}\text { Características } \\
\text { del paciente }\end{array}$ & $\begin{array}{l}\text { Características } \\
\text { del episodios }\end{array}$ & $\begin{array}{l}\text { Episodios } \\
\text { de recurrencia }\end{array}$ & $\begin{array}{l}\text { Tiempo desde el primer } \\
\text { episodio hasta el desarrollo } \\
\text { de la MNVSR }\end{array}$ & $\begin{array}{l}\text { Agudeza visual } \\
\text { final }\end{array}$ \\
\hline $\begin{array}{l}\text { Wyhinny GJ } \\
\text { et al }\end{array}$ & $\begin{array}{l}32 \text { años } \\
\text { Mujer } \\
\text { Infección del tracto } \\
\text { respiratorio superior } \\
1 \text { semana antes }\end{array}$ & $\begin{array}{l}\text { Visión borrosa } \\
\text { ojo izquierdo } \\
\text { Agudeza Visual 20/60 } \\
\text { Resolución del episodio } \\
\text { en } 1 \text { mes sin secuelas, } \\
\text { agudeza visual final 20/25 } \\
\text { Nervio óptico hiperémico } \\
\text { e inflamado }\end{array}$ & $\begin{array}{l}\text { Recurrencia } \\
\text { a los } 4 \text { meses }\end{array}$ & $\begin{array}{l}6 \text { meses } \\
\text { agudeza visual 20/80 } \\
\text { MNVSR* subfoveal } \\
\text { No tratamiento }\end{array}$ & $20 / 40$ \\
\hline $\begin{array}{l}\text { Mc Collum CJ } \\
\text { et al }\end{array}$ & $\begin{array}{l}27 \text { años } \\
\text { Mujer } \\
\text { Varicela } 10 \text { días } \\
\text { antes }\end{array}$ & $\begin{array}{l}\text { Visión borrosa y escotoma } \\
\text { central ojo izquierdo } \\
\text { Agudeza Visual 20/50 } \\
\text { Resolución del episodio } \\
\text { en } 8 \text { semanas, agudeza } \\
\text { visual final 20/30 } \\
\text { Manchas blancas y cambios } \\
\text { pigmentarios peripapilares }\end{array}$ & $\begin{array}{l}\text { Recurrencia al año } \\
\text { Agudeza visual } \\
20 / 50\end{array}$ & $\begin{array}{l}\text { MNVSR* peripapilar } \\
\text { en supero-nasal }\end{array}$ & $\begin{array}{l}\text { No tratamiento } \\
20 / 30\end{array}$ \\
\hline $\begin{array}{l}\text { Oh KT } \\
\text { et al }\end{array}$ & $\begin{array}{l}36 \text { años } \\
\text { Mujer } \\
\text { Infección } \\
\text { respiratoria } \\
\text { previa }\end{array}$ & $\begin{array}{l}\text { Escotoma central y } \\
\text { fotopsias ojo derecho } \\
\text { Agudeza Visual 20/40 } \\
\text { Presentaba defecto pupilar } \\
\text { aferente relativo } \\
\text { Resolución del episodio en } \\
2 \text { semanas, agudeza visual } \\
\text { final 20/30 } \\
\text { Atrofia peripapilar }\end{array}$ & $\begin{array}{l}\text { Recurrencia en ojo } \\
\text { contralateral a los } \\
9 \text { años }\end{array}$ & $\begin{array}{l}13 \text { años desde el primer } \\
\text { episodio } \\
\text { MNVSR* ojo derecho } \\
\text { afectación peripapilar } \\
\text { y macular }\end{array}$ & $20 / 250$ \\
\hline Low V & $\begin{array}{l}48 \text { años } \\
\text { et al }\end{array}$ & $\begin{array}{l}\text { Visión borrosa y aumento } \\
\text { de la mancha ciega }\end{array}$ & & $\begin{array}{l}4 \text { semanas tras el inicio } \\
\text { de primer episodio } \\
\text { Terapia fotodinámica }\end{array}$ & \\
\hline $\begin{array}{l}\text { Figueroa MS } \\
\text { et al }\end{array}$ & $\begin{array}{l}46 \text { años } \\
\text { Mujer } \\
\text { No refiere episodio } \\
\text { gripal previo }\end{array}$ & $\begin{array}{l}\text { Disminución de la visión } \\
\text { Vitritis } \\
\text { Edema de papila } \\
\text { Edema macular }\end{array}$ & $\begin{array}{l}\text { Nueve episodios en } \\
\text { siete años, tres de } \\
\text { ellos bilaterales }\end{array}$ & $\begin{array}{l}2 \text { años después del } \\
\text { primer brote } \\
\text { NVC** peripapilar bilateral } \\
\text { Fotocoagulación láser }\end{array}$ & \\
\hline
\end{tabular}

* MNVSR: membrana neovascular subretiniana; ** NVC: neovascularización coroidea.

con MEWDS recurrente y habitualmente bilateral, lesiones focales de atrofia coriorretiniana localizadas próximas a papila, en polo posterior y media periferia con cierto parecido a las lesiones de $\mathrm{CMF}$ y presunta histoplasmosis ocular, aunque con un patrón de cicatrización distinto. Todo esto nos inclina a pensar en un probable MEWDS atípico. Bryan et al (5), describen una serie de casos de MEWDS previo o tras una CMF, probablemente exista una susceptibilidad, etiología y/o patogenia comunes a estas dos entidades clínicas, aunque esto aun se desconoce.

A pesar del buen pronóstico visual de los pacientes con MEWDS, creemos que estos pacientes deberían ser evaluados a largo plazo por el riesgo de aparición de MNVSR.

\section{BIBLIOGRAFÍA}

1. Oh KT, Christmas NJ, Russel SR. Late recurrence and choroidal neovascularization in multiple evanescent white dot syndrome. Retina 2001; 21: 182-184.

2. Tsai L, Jampol LM, Pollock SC, Olk J. Chronic recurrent multiple evanescent white dot syndrome. Retina 1994; 14: 160-163.

3. Figueroa MS, Ciancas E, Mompean B, Quereda C. Treatment of multiple evanescent white dot syndrome with cyclosporine. Eur J Ophthalmol 2001; 11: 86-88.

4. Kozielec GF, Wyhnny GJ, Jampol LM. Evolution of distinct chorioretinal scars in recurrent MEWDS. Retina 2001; 21: 180-182.

5. Bryan RG, Freund KB, Yannuzzi LA, Spaide RF, Huang SJ, Costa DL. Multiple evanescent white dot syndrome in patients with multifocal choroiditis. Retina 2002; 22: $317-$ 322. 